\title{
Design Development and Characterisation of Tramadol Hydrochloride Loaded Transethosomal Gel Formulation for Effective Pain Management
}

\author{
Vankayala Devendiran Sundar*, Pamu Divya, Magharla Dasaratha Dhanaraju \\ Department of Pharmaceutical Technology, GIET School of Pharmacy, Rajahmundry, Andhra Pradesh, INDIA.
}

\begin{abstract}
Introduction: The purpose of the present study was to design develop and characterize the tramadol hydrochloride loaded transethosomal gel formulation for effective pain management. Materials and Methods: The transethosomes were prepared by simple cold method. Total 12 formulations were prepared using different concentrations of phospholipid (Soya lecithin and L- $\alpha$ Phosphatidylcholine from egg yolk) and edge activator (Span 20 and Cremophor EL 35).The developed transethosomes were characterized for FTIR, drug content, EE, particle size, zeta potential, TEM, in vitro drug release and release kinetics. The optimised formulation was selected for preparation of transethosomal gel. The formulated gel was estimated for viscosity, drug content, EE and stability study for 28 days. Results and Discussion: The zeta potential of best formulation was $-22 \mathrm{mV}$, the particle size was at the range of $149.34 \mathrm{~nm}$ to $278 \mathrm{~nm}$. Span 20 formulations exhibit a faster drug release (91.91to $95.7 \%$ ) than the Cremophor formulations, whose release exhibits an extended pattern $(78.96 \%$ to $79.34 \%)$ at the end of $8^{\text {th }}$ hr. Optimised formulation follows first order kinetics and its $\mathrm{R} 2$ value is 0.991 . Conclusion: The study supports the development of optimised transethosome formulation into a topical gel using carbopol 934 as gelling agent. The viscosity of the gel formulation was $30168 \mathrm{cps}$. The drug content and EE was found to be $91.52 \%$ and $79.37 \%$ respectively. Stability studies prove that there is very less change in the $E E$, hence the formulation was found to be stable.
\end{abstract}

Key words: Transethosomes, Tramadol hydrochloride, Phospholipid, Edge activator, Entrapment efficiency.

\section{INTRODUCTION}

Topical drug delivery of active pharmaceutical carriers, which a drug is delivered in the targeted area under controlled system can have a significant effect on efficacy. ${ }^{1}$ The main advantage of the topical drug delivery is to bypass first pass metabolism. Topical drug carriers are used for the localized effect of application and deliver the drug into underlying percutaneous layers of the skin. ${ }^{2}$ These systems can be characterized as controlled and targeted drug delivery systems. The systems raise the efficacy of the drug and dominate the side effects for the improved patient compliance. The polardrug molecules are encapsulated into the hydrophobic channel carrier which is prevented from hydrolysis and enzymatic degradation ${ }^{3,4}$ The ethosomal carriers are vesicular systems that comprise phospholipids, water and a moderately increased concentration of ethanol. Ethosomal systems are of three type's namely classical ethosomes, binary ethosomes and transethosomes (TEs). ${ }^{5}$ These are of novel generation and developed in 2012. ${ }^{6}$ They usually comprises of ethosomes and an edge activator or a penetration enhancer. The transethosomes improves the physical and chemical qualities of the therapeutic drug enclosed in dermal
Submission Date: 20-07-2019; Revision Date: 06-02-2020; Accepted Date: 12-03-2020

DOI: 10.5530/ijper.54.2s.65 Correspondence:

Dr. Vankayala Devendiran Sundar

Professor, Department of Pharmaceutical Technology, GIET School of Pharmacy, $\mathrm{NH}-16$, Chaitanya Knowledge City, Rajahmundry-533296, Andhra Pradesh, INDIA.

Phone: +919959212600 E-mail: sundarvd@yahoo. co.in

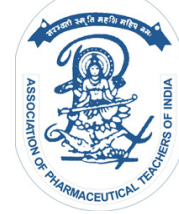

www.ijper.org 
and transdermal delivery. The effect of enhancement is proven by several researchers. ${ }^{6-8}$ These transethosomes contain high concentration of ethanol which induces hydration of the outer layer of the skin and widen the pores and initiate the drug release to the targeted site. The transethosomes are prepared by using cold method. ${ }^{910}$ The cold method is simple and widely used method for the preparation of transethosomes. Tramadol hydrochloride is a centrally acting analgesic which is used to treat mild pain to severe pain. The drug is recommended for nociceptive pain commonly responsive to anti-inflammatory agents and opiates. Tramadol hydrochloride shows high solubility and permeability which increase its concentration in the central nervous system. Tramadol hydrochloride loaded transethosomal topical gel formulation reduces the adverse effects of the drug and increases the therapeutic efficacy. Transethosomes penetrate and target the stimulation of specialized nerve endings pain receptors which sense and respond to the damaged nerves. Phospholipids from different sources have been used in the transethosomal system, the type of phospholipid used influences the vesicle formation of the transethosomes. ${ }^{11,12}$ The L- $\alpha$ phosphatidylcholine from egg yolk lyophilized powder and soya lecithin is used as encapsulating agent in the preparation of transethosomes. ${ }^{13}$ The type of edge activator used has a significant role in the size, stability and used to increase the penetration of active drug into the skin. The selection of the edge activator is the critical step in formulation of the transethosomes. Span 20 and Cremophor EL35 were used as edge activators. The incorporation of edge activators prevents the leakage of the vesicles and maintains the vesicular charge. Ethanol is the solvent system used as the penetration enhancer which helps in fluidization of the skin stratum corneum and helps the release of the drug to the underlying layers of the skin. These transethosomal systems were incorporated into suitable gelling systems which are compatible and provide required viscosity and bio adhesive properties. ${ }^{14}$ Incorporating into the gelling agent results in controlled release of the drug and prevent the loss of the drug from the transethosomal vesicles. This advance method of formulation of transethosomes helps to cutdown the side effects of the orally consumed drugs for minor pains and topically deliver the drug to effected targeted area in a controlled system. The focus of the present study is to develop and characterize transethosomal gel carriers for topical delivery of tramadol hydrochloride as a route for the drug administration. In this study tramadol hydrochloride encumbered TEs were prepared with various concentrations of solvent system and edge activator. The optimised formulation was incorporated into a gel base.

\section{MATERIALS AND METHODS}

Tramadol hydrochloride was collected gifted sample from Aurobindo Pharma Pvt Ltd, Hyderabad. Span 20, Cremophor EL-35 was obtained from Lobachem. Soya lecithin and L-aphosphatidylcholine from egg yolk lyophilized were purchased from Sigma - Aldrich, Mumbai. Carbopol 934 was obtained from CDH, New Delhi. The chemicals utilized in the research are of AR grade were procured from Merck, Mumbai.

\section{Preparation of tramadol hydrochloride loaded TEs}

The cold method was used for the preparation of tramadol hydrochloride transethosomes. A solution of phosphatidylcholine and surfactant (Span20, Cremophor EL-35) in ethanol at $30^{\circ} \mathrm{C}$ act as organic phase. The aqueous phase / ultra pure water was heated at $30^{\circ} \mathrm{C}$ and then delivered to the organic phase drop wise with constant mixing at $700 \mathrm{rpm}$ with the help of magnetic stirrer. Continue the stir for $45 \mathrm{~min}$ to bring the transethosomal dispersions that were supplemented for size-reduction by probe sonication for 10 mins at room temperature. The method was found to give more stable transethosomes. The composition of these formulations was shown in Table 1.

\section{Evaluation of Transethosomes}

\section{Drug Excipient compatibility study}

The drug interaction and the composition compatibility within the formulation ingredients were determined using FT-IR analysis. The IR spectrum was performed by using a (Bruker FTIR) spectrophotometer. The samples were recorded using the conventional $\mathrm{KBr}$ pellet method. The samples were scanned in the region between 4000 and $400 \mathrm{~cm}^{-1}$ with a resolution of about $4 \mathrm{~cm}^{-1}$.

\section{Assay}

The drug content in the developed transethosome formulation was determined. An amount of transethosomes containing tramadol hydrochloride equivalent to $100 \mathrm{mg}$ were weighed and suspended in suitable media. This was conserved for about $20 \mathrm{~min}$ for thorough mixing. Agitate continuously and filter by $0.45 \mu \mathrm{m}$ membrane filter. The amount of tramadol present was estimated at $271 \mathrm{~nm}$ using a regression equation through the linearity plot. ${ }^{15}$

Drug content $=$ Sample absorbance $/$ Standard absorbance $\times 100$ 


\section{Percentage drug EE}

The EE was estimated using mini column centrifugation method. The samples were wrecked to centrifugation at $8000 \mathrm{rpm}$. The supernatant was discarded the obtained sediment was dissolved in mixture of dichloromethane and methanol. The sediment was suspended in suitable media and the absorbance was taken at $271 \mathrm{~nm} .{ }^{16,17}$ The drug $\mathrm{EE}$ was quantified by applying the below equation EE \% = Amount of entrapped drug / Total amount of drug $\times 100$

\section{Evaluation of vesicle size}

Vesicle size was assessed by DLS (dynamic light scattering) using a programmed scrutiny system and Photon Correlation Spectroscopy (PCS). The size of transethosomes ranges between nanometres and is influenced by the composition of the formulation. Size and size distribution of transethosomes were carried out using Beckman coulter particle size analyser (Delsa ${ }^{\mathrm{TM}}$ Nano Common). The transethosome was distributed in double distilled water prior to administration of the sample in the instrument, to establish that the signal of light scattering, as indicated by particle count/second was in the instrument's sensitivity range. ${ }^{18}$

\section{Transethosomes Morphology}

Shape and morphology of the TEs was investigated using transmission electron microscopy. Anaqueous solution of $2 \% \mathrm{w} / \mathrm{v}$ phosphotungstic acid on a copper grid (carbon-coated) was used to stain negatively the prepared transethosomes and was examined by TEM (FEI Tecnai G2 F20 S-twin/EDAX). The prepared transethosome was dropped onto the carbon coated grid; the additional solution was extracted using a blotting paper. Allow the grid to dry for 5 min. ${ }^{18}$ The TEM micrograph was taken by applying an accelerating voltage of $200 \mathrm{kV}$.

\section{Zeta potential}

The surface charge of TEs was estimated by conducting Zeta potential measurements. The estimation was conducted in distilled water with conductivity regulated to $0.0504 \mathrm{~ms} / \mathrm{cm}$. Zeta potential was checked in the original dispersion medium (water). ${ }^{19}$ The analysis was performed using the Beckman Coulter (Delsa ${ }^{\mathrm{TM}}$ Nano Common).

\section{In vitro drug release}

The release studies were estimated by Franz diffusion cell. A cellophane dialysis membrane (Hi-media 60) was hydrated with phosphate buffer ( $\mathrm{pH}$ 7.4). Two millilitres of the TEs equivalent to $100 \mathrm{mg}$ of tramadol hydrochloride was kept in donor compartment. ${ }^{17}$ The receptor compartment was replete with $\mathrm{pH} 7.4$ and mixed with a magnetic bar at $500 \mathrm{rpm}$ at $37 \pm 1^{\circ} \mathrm{C}$. The required sample was taken off at predetermined time intervals for $8 \mathrm{~h}$ and was assessed for tramadol hydrochloride content by UV spectrophotometrically at $271 \mathrm{~nm}$.

\section{Analysis of release mechanism}

The release mechanisms and the design of therapeutic device mathematical models are widely employed. The study of in vitro release profile of formulations was done by implementing appropriate release data in several drug release models including zero order, first order and Higuchi. The mentioned models are well known for the characterization drug dissolution / release profile.

\section{Preparation of tramadol hydrochloride loaded TEs gel}

Tramadol hydrochloride-loaded TEs gel was prepared by including the requisite quantity of the optimized tramadol hydrochloride enclosed TEs in a previously

\begin{tabular}{|c|c|c|c|c|c|c|c|c|c|c|c|c|}
\hline Ingredients & F 1 & F2 & F 3 & F 4 & F 5 & F 6 & F7 & F8 & F9 & F10 & F11 & F 12 \\
\hline Tramadol (mg) & 100 & 100 & 100 & 100 & 100 & 100 & 100 & 100 & 100 & 100 & 100 & 100 \\
\hline Soya lecithin (SC) & $2 \%$ & $3 \%$ & $5 \%$ & - & - & - & $2 \%$ & $3 \%$ & $5 \%$ & - & - & - \\
\hline $\begin{array}{c}\text { I- } \alpha \\
\text { Phosphatidylcholine } \\
\text { from egg yolk }(\mathrm{PL})\end{array}$ & - & - & - & $2 \%$ & $3 \%$ & $5 \%$ & - & - & - & $2 \%$ & $3 \%$ & $5 \%$ \\
\hline Span 20 & $20 \%$ & $40 \%$ & $50 \%$ & $20 \%$ & $40 \%$ & $50 \%$ & & & & & & \\
\hline Cremophor EL-35 & & & & & & & $0.3 \%$ & $0.5 \%$ & $1 \%$ & $0.3 \%$ & $0.5 \%$ & $1 \%$ \\
\hline Ethanol & $20 \%$ & $30 \%$ & $45 \%$ & $20 \%$ & $30 \%$ & $45 \%$ & $20 \%$ & $30 \%$ & $45 \%$ & $20 \%$ & $30 \%$ & $45 \%$ \\
\hline Distilled water (qs) & $100 \%$ & $100 \%$ & $100 \%$ & $100 \%$ & $100 \%$ & $100 \%$ & $100 \%$ & $100 \%$ & $100 \%$ & $100 \%$ & $100 \%$ & $100 \%$ \\
\hline
\end{tabular}


made gel base at the proportion of $4: 1$ by slow mixing, followed by manual stirring for $10 \mathrm{~min}$. Disperse Carbopol 934 in distilled water and leave to swell overnight which is used as base for preparation of gel. Propylene glycol is used to dissolve methyl paraben and propyl paraben. The solution is mixed to get the gel mixture. Neutralisation of prepared gel was achieved by adding triethanolamine by frequent stirring until $\mathrm{pH}$ (7.0) was obtained and to finally get the consistent translucent gel base. ${ }^{19}$

\section{Characterization of the tramadol hydrochloride loaded transethosomal gel}

\section{Determination of $\mathrm{pH}$}

Digital pH meter (Elico LI610) was used to estimate the $\mathrm{pH}$ of the gel. A known quantity of transethosomal gel was disseminated in $20 \mathrm{ml}$ of distilled water and mixed for $30 \mathrm{~min}$ by a mechanical stirrer set to room temperature; the $\mathrm{pH}$ of the formulation was read from the digital display.

\section{Viscosity, drug content and EE}

Brookfield CAP 2000 viscometer was used to determine the viscosity of tramadol hydrochloride TEs gel using spindle no 16 at $25^{\circ} \mathrm{C}$. Drug content was estimated by weighing an amount of transethosomes gel containing tramadol hydrochloride equivalent to $100 \mathrm{mg}$ was suspended in suitable media. The final dispersion was kept for $20 \mathrm{~min}$ for thorough mixing with often frequent mixing and then it was filtered through $0.45 \mu \mathrm{m}$ membrane filter. The drug content/amount was assessed at $271 \mathrm{~nm}$ spectrophotometrically using a regression equation of the Beer's Law plot. ${ }^{19}$

\section{Stability Studies}

Drug EE of transethosomal gel containing $100 \mathrm{mg}$ of drug was taken and was dissolved in mixture of DCM (Dichloromethane) and methanol. The dissolved mixture was suspended in $100 \mathrm{ml}$ of phosphate buffer (pH 7.4) and the absorbance was noted at $271 \mathrm{~nm}$. From that the amount present in $1 \mathrm{ml}$ of transethosomes was obtained. The drug EE was estimated by the formula used in TEs.

The stability studies of tramadol hydrochloride TEsloaded formulation were performed by keeping freshly made samples of the gel in two sets storage conditions namely at room temperature $25 \pm 2^{\circ} \mathrm{C}$ and refrigerated at $5 \pm 3^{\circ} \mathrm{C}$ and for 28 days. ${ }^{15,20}$ The stored transethosome gel was evaluated for percentage drug content and EE at an interval of 7 days for 28 days.

\section{RESULTS AND DISCUSSION}

\section{Transethosomes loaded with tramadol hydrochloride}

The tramadol hydrochloride encapsulated transethosomes were formulated for effective pain management by cold method using different lipoidal carrier systems. The cold method was followed for the preparation of TEs as it is very simple and which does not require extreme conditions or sophisticated equipment. Total 12 formulations were developed by using cold method. Formulations were designed using ethanol, edge activator and phospholipid. Span 20 and Cremophor EL-35 were used as edge activators, were as soya lecithin and 1- $\alpha$ Phosphatidylcholine from egg yolk contributes to lipid carrier and ethanol as solvent system. The size of vesicle is an significant factor that should be consider in the development of Nano carriers for topical drug delivery. ${ }^{21}$ Ethanol is a major component of the ethosomal systems, forgiving exceptional characteristics. $10 \%-50 \%$ is the concentration commonly used. The phospholipid concentration ranged between 0.5 and $5 \% \mathrm{w} / \mathrm{w}^{22}$ The formulations were developed with the recommended concentration of phospholipid and ethanol.

\section{Drug Excipient compatibility study}

Figure 1 epic the FTIR absorption spectrum of pure drug tramadol hydrochloride, which was taken in the range of $700 \mathrm{~cm}^{-1}-4000 \mathrm{~cm}^{-1}$ using $\mathrm{KBr}$ disc and the characteristic peak of the drug was observed. The IR spectra of developed formulation and physical mixture and were studied, the results reveals that the characteristic peak of pure drug was predominantly present, which proves the drug and excipients are compatible. No change in shape of spectra observed, indicating that the drug in

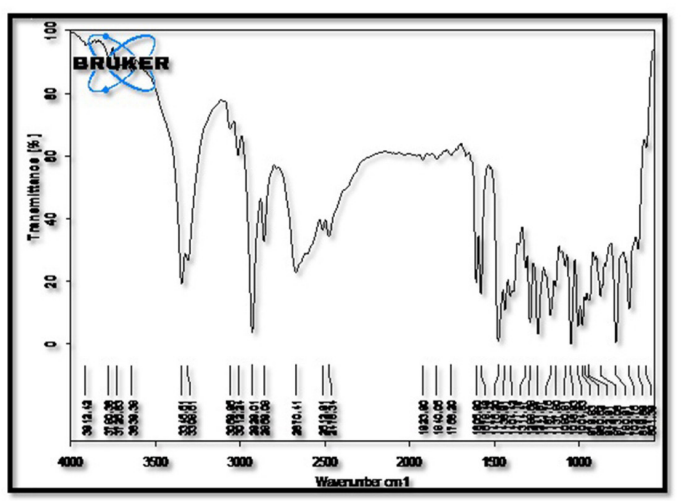

Figure 1: FTIR absorption spectrum of pure drug tramadol hydrochloride. 
presence of excipients remains stable. The results were shown in Figure 2a and 2b.

\section{Assay}

The quantity of drug content in the developed transethosome formulation were determined and estimated by UV spectrophotometry at $271 \mathrm{~nm}$. The amount of tramadol hydrochloride in transethosomal formulations was found to be in the range of 91.91 to $95.7 \%$. Among all the formulations F8 was found to be $95.24 \%$. The results of drug content were given in Table 2.

\section{Percentage drug EE}

The EE of the developed formulations were in the range of $33.43 \%$ to $79.43 \%$. The formulations F1 - F3 comprise of Soya lecithin, Span 20 and ethanol as the solvent. Formulation F2 showed higher EE $60.21 \%$ and F3 exhibit low EE $33.43 \%$. The effect of variation in entrapment is the presence of ethanol concentration in formulation. Higher concentration of ethanol in the formulation induces the vesicle solubility which leads to leakage of the transethosomal vesicles. The formulation F4 - F6 comprising of L- aphosphatidylcholine

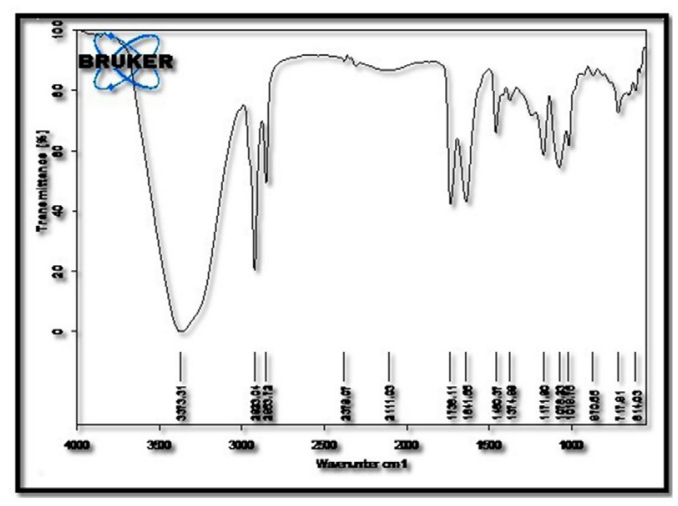

Figure 2a: IR spectra of physical mixture pure drug and excipients.

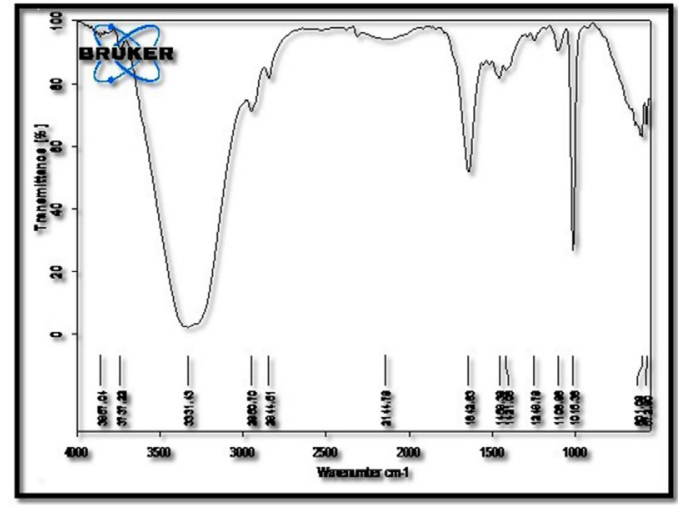

Figure 2b: IR spectra of optimised transethosome formulation. from egg yolk, Span 20 and ethanol. Among the formulations F5 showed higher EE63.56\% because of optimum concentration ethanol. The formulations F7 - F9 comprising Cremophor EL-35, soya lecithin and ethanol. Formulation F10 - F12 comprising of L- aphosphatidylcholine from egg yolk, Cremophor EL-35 and ethanol as a solvent system. The formulation F7 - F12 was developed with the change in the edge activator Cremophor EL- 35 lead to higher EE. The concentration of the edge activator has a significant role on the transethosome EE. The formulation F8 and F11 showed higher EE78.91\% and $79.43 \%$ where minimal concentration of ethanol enhances EE. Higher concentration of ethanol in the formulation that induces vesicular solubility which leads to leakage and lower the EE. ${ }^{23}$ The results were given in Table 2.

\section{Evaluation of vesicle size}

The size of vesicles plays an essential role in skin permeation. Smaller vesicular size $(<200 \mathrm{~nm})$ ease the TEs to diffuse by the tiny pores of the skin giving rise to improved permeation. The size was determined by DLS (dynamic light scattering) using a computerized inspection system and photon correlation spectroscopy. The formulation exhibits an average particle size in the range of $149.34 \mathrm{~nm}$ to $198.10 \mathrm{~nm}$ and the results were shown in Figure 3. The vesicle size of formulations was influenced by the polymer concentration, as the concentration increases the formulations exhibits an increase in vesicle size. Higher concentration of the phospholipids and edge activator produces thick vesicles with high dense and thus increase the size.

\begin{tabular}{|c|c|c|c|}
\multicolumn{4}{|c|}{ Table 2: Drug content, EE and zeta potential of TEs } \\
Formulation. \\
$\begin{array}{c}\text { Formulation } \\
\text { Code }\end{array}$ & $\begin{array}{c}\text { Drug content } \\
\text { (\%) } \\
\mathbf{I S D}\end{array}$ & $\mathbf{E E ( \% ) \pm S D}$ & $\begin{array}{c}\text { Zeta } \\
\text { potential } \\
\mathbf{\pm S D}\end{array}$ \\
\hline F1 & $91.91 \pm 0.03$ & $46.45 \pm 0.213$ & $-7.76 \pm 0.041$ \\
\hline F2 & $93.97 \pm 0.23$ & $60.21 \pm 0.034$ & $-9.99 \pm 0.053$ \\
\hline F3 & $94.38 \pm 0.041$ & $33.43 \pm 0.151$ & $-7.48 \pm 0.026$ \\
\hline F4 & $81.32 \pm 0.02$ & $48.37 \pm 0.432$ & $-17.83 \pm 0.028$ \\
\hline F5 & $90.99 \pm 0.05$ & $63.56 \pm 0.031$ & $-21.49 \pm 0.043$ \\
\hline F6 & $92.97 \pm 0.04$ & $50.70 \pm 0.313$ & $-18.59 \pm 0.15$ \\
\hline F7 & $93.24 \pm 0.12$ & $78.91 \pm 0.053$ & $-11.53 \pm 0.16$ \\
\hline F8 & $95.39 \pm 0.03$ & $78.96 \pm 0.041$ & $-19.87 \pm 0.091$ \\
\hline F9 & $89.92 \pm 0.41$ & $68.78 \pm 0.036$ & $-17.68 \pm 0.084$ \\
\hline F10 & $92.31 \pm 0.32$ & $77.370 \pm 0.063$ & $-20.61 \pm 0.073$ \\
\hline F11 & $93.56 \pm 0.25$ & $79.43 \pm 0.121$ & $-22.30 \pm 0.082$ \\
\hline F12 & $95.70 \pm 0.13$ & $76.45 \pm 0.0143$ & $-18.30 \pm 0.043$ \\
\hline
\end{tabular}


The size of the vesicles was further influenced by the type of phospholipid used in the development of formulation. The vesicle size was increased due to increased fusion of lipid bilayer and consequent increase in surface free energy that ultimately occur by the influence of density of lipids. ${ }^{20}$ Formulation developed using soya lecithin exhibits an increased particle size than egg yolk formulations. Soya lecithin formulations are viscous than the 1- $\alpha$ Phosphatidylcholine from egg yolk formulations.

The presence of the edge activator plays significant role in vesicular size. Formulation using Span 20 exhibits lager vesicular size than Cremophor EL- 35. As the concentration of edge activator increases, the formulation exhibits a decrease in vesicular size.

\section{Transethosomes Morphology}

The morphology of the TEs formulation was visualized by the TEM. The TEs formulations had asymmetrical shape. This might be ascribed to the presence of the edge activator (Cremophor EL-35 and Span 20) in the composition of the vesicles which flustered the lipid

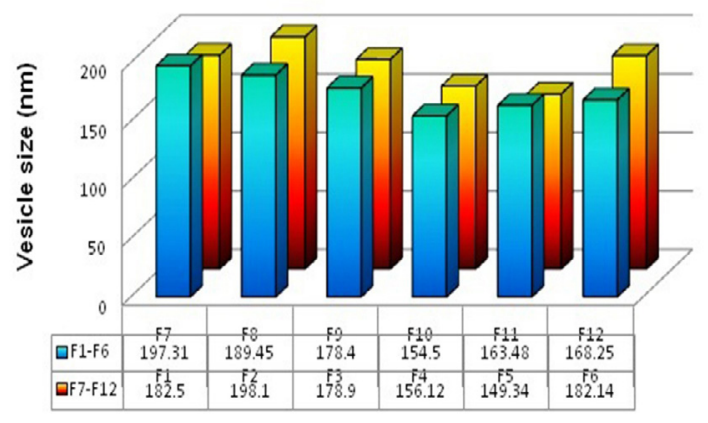

Figure 3: Vesicle Size of Transethosome Formulations F1 - F12.
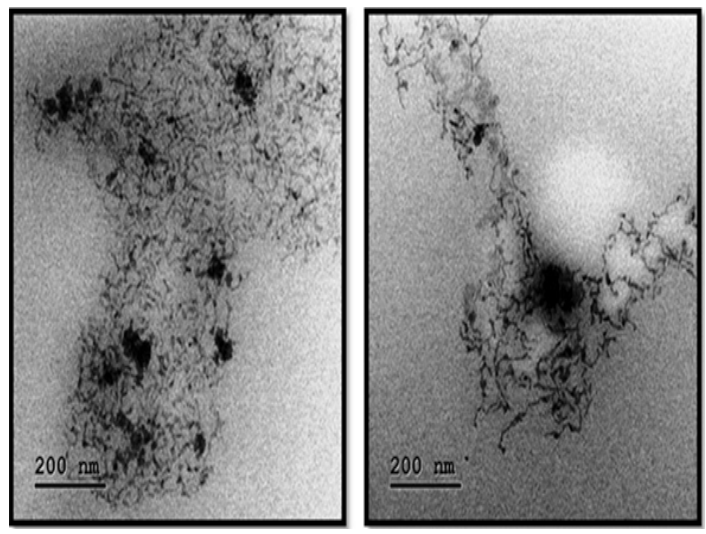

Figure 4: TEM of the Transethosome Formulation Containing Edge Activator Span 20. bilayer and raised their fluidity. ${ }^{6}$ The TEM of tramadol loaded transethosomes was shown in Figure 4 and 5.

\section{Zeta potential}

The zeta potential analysis was performed to investigate the surface charge properties of TEs. The transethosome stability and interaction of formulation with skin was influenced by zeta potential. Presence of ethanol in formulation exhibits a negative charge on the polar groups of phospholipids that build an electrostatic repulsion. The zeta potential of the transethosomes was found between the ranges of $-7.76 \mathrm{mV}$ to $-22.30 \mathrm{mV}$. The zeta potential peak of charge $-22 \mathrm{mV}$ was shown in Figure 6. Thus, it was concluded that the TEs system had sufficient stability. The results were given in Table 2 .

\section{In vitro drug release}

The in vitro diffusion studies were performed for the prepared TEs formulations by using Franz diffusion cell. The results of diffusion studies conducted for TEs formulations containing tramadol hydrochloride were reported in Figure $7 \mathrm{a}$ and $7 \mathrm{~b}$.

\section{Effect of Edge Activator}

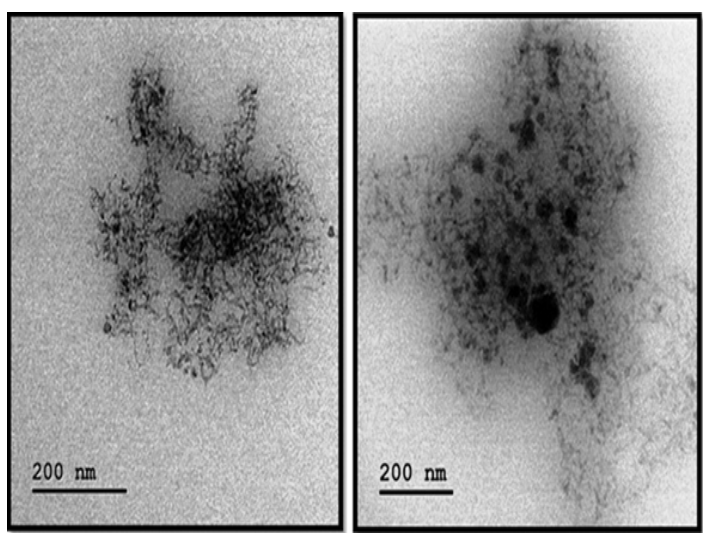

Figure 5: TEM of the Transethosome Formulation Containing Edge Activator Cremophor EL-35.

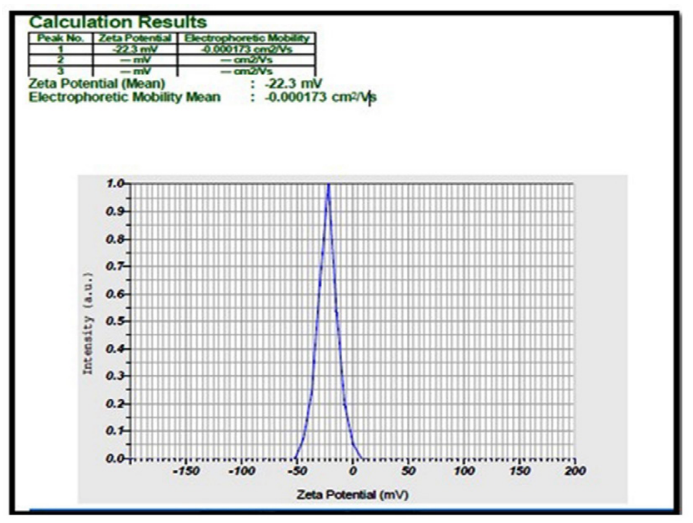

Figure 6: Zeta Potential of optimised transethosome formulation. 
The use of edge activator in formulation increases the skin permeability and there which increases the release of tramadol. The in vitro release of transethosomes prepared by Span 20 as edge activator exhibits a higher rate of release than Cremophor EL-35. Cremophor EL-35 formulations exhibits controlled release over span, the tendency is due to viscous nature of edge

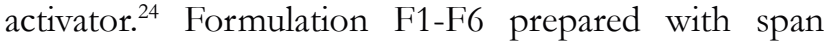
exhibit a drug release of $90.12 \%$ to $94.99 \%$ which is higher than cremophor formulations (F7- F12, 88.34\% to $82.19 \%$ ) at the end of $8^{\text {th }} \mathrm{hr}$.

\section{Effect of Ethanol}

The formulations were developed using increase concentration of ethanol as permeation enhancer. Ethanol is an efficient penetration enhancer; the concentration of ethanol influences the solubility of lipids. ${ }^{25}$ As the concentration of ethanol increases, lipid vesicle miscibility increases. Among the developed formulations, those prepared with $40 \%$ exhibits a rapid release than the other at the end of $8^{\text {th }} \mathrm{hr}$. Formulation F8 and F11 prepared with $30 \%$ of ethanol as solvent exhibits

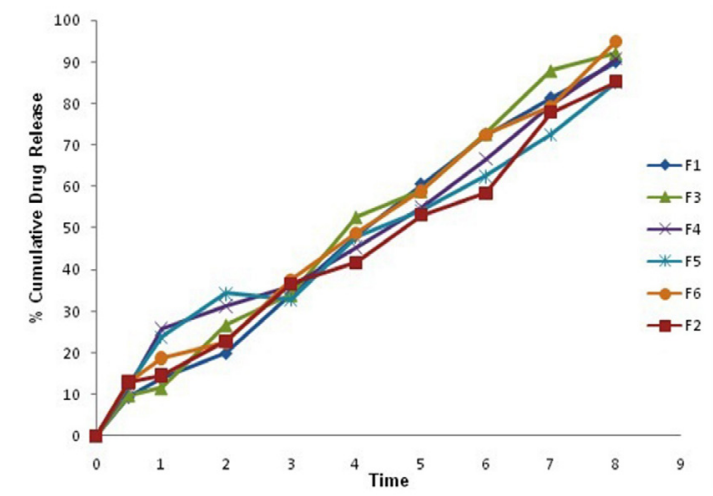

Figure 7a: Cumulative \% Drug release of TEs formulations F1-F6.

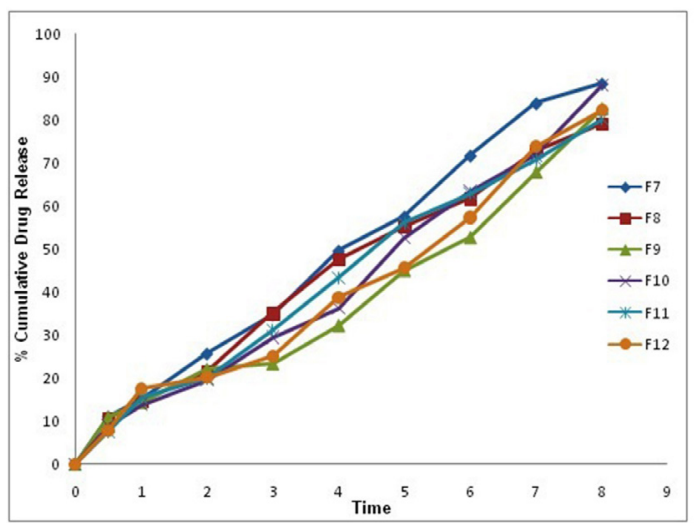

Figure 7b: Cumulative \% Drug release of TEs formulations F7-F12. a controlled drug release of $78.96 \%$ and $79.98 \%$ at the end of $8^{\text {th }}$ h. An optimized concentration proves to be controlled.

\section{Effect of Phospholipid}

The phospholipid bilayer in vesicular formulation can influence both encapsulation and release of the drug particles. The nonpolar hydrophobic part of the phospholipid has more affinity towards the skin thus fuses with the lipid content of the skin and facilitates the delivery of the entrapped material. The formulation was developed by using soya lecithin and egg yolk as phospholipid. The phospholipid was used at a concentration of $2 \%, 3 \%$ and $5 \%$. Formulation developed with higher concentration of phospholipid exhibits a faster release than with the lower concentration with $3 \%$ due to greater affinity towards the skin. ${ }^{25,26}$

Among all formulations, F11 comprising ethanol 30\% and $2 \%$ of phospholipid and suitable edge activator (Cremophor EL-35) has found to be the optimum batch which exhibits a controlled release of tramadol hydrochloride from the TEs formulation at the end of $8^{\text {th }} \mathrm{hr}$. Further the formulation F11 is considered for the development of transethosomal gel formulation.

\section{Analysis of release mechanism}

The release data of tramadol hydrochloride transethosome formulation F11 was fitted into various kinetic models such as zero order, first order and highchair in order to analyse the mechanism of release of tramadol hydrochloride from TEs. The release pattern of the formulation F11 exhibit best fit of highest correlation coefficient in First order kinetics $R^{2}$ value 0.991 . Hence the formulation follows diffusion followed by dissolution pattern.

\section{Characterization of the tramadol hydrochloride loaded transethosomal gel}

The cold method was followed for the making of tramadol hydrochloride transethosomes. Among the prepared formulations the optimised batch was incorporated into carbopol 934 gel base. The prepared tramadol hydrochloride gel was further characterised for $\mathrm{pH}$, viscosity, drug content and EE. The results of characterization of the tramadol hydrochloride-loaded transethosomal gels are briefed in Table 3 .

The $\mathrm{pH}$ of the preparation was found to be 7.0; the transethosome gel formulation was evaluated for viscosity, drug content and $\mathrm{EE}$. The viscosity of the transethosomal gel formulation was found to be 30168 cps, the drug content and EE of transethosomal gel was found to be 91.52 and $79.37 \%$ respectively. 


\section{Table 3: Characterisation of TEs gel formulation.}

\begin{tabular}{|c|c|c|c|c|}
\hline Formulation & pH & Viscosity & $\begin{array}{c}\text { Drug } \\
\text { Content }\end{array}$ & EE \\
\hline $\begin{array}{c}\text { Transethosomal } \\
\text { gel formulation }\end{array}$ & 7.0 & $30168 \mathrm{cps}$ & $91.52 \%$ & $79.37 \%$ \\
\hline
\end{tabular}

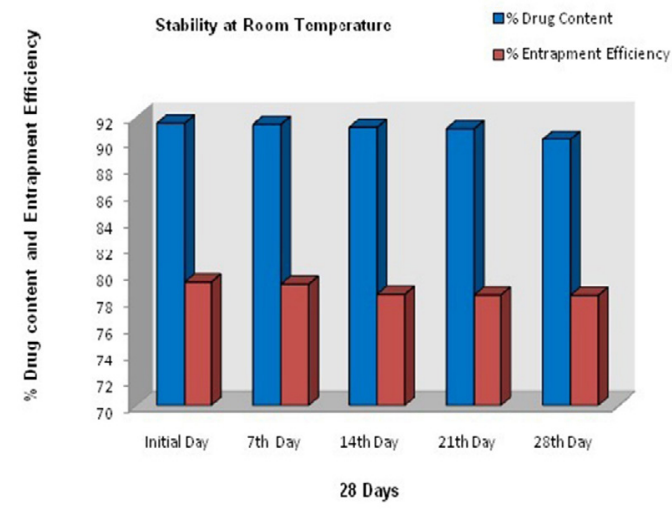

Figure 8: Depicting the stability study of transethosome gel at refrigerated condition.

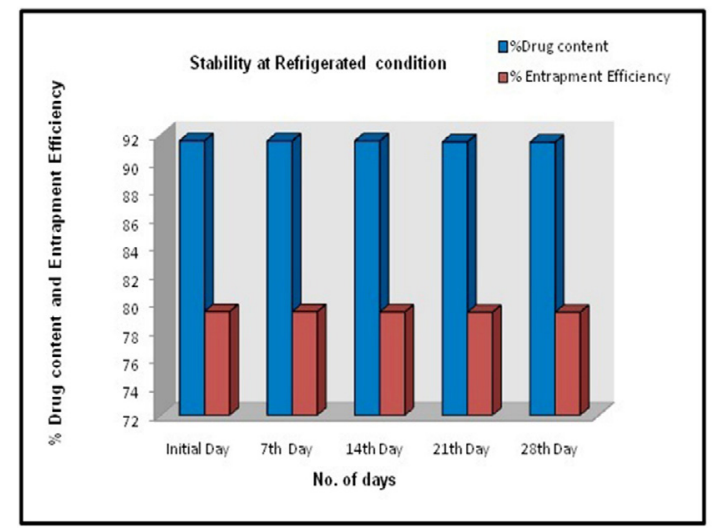

Figure 9: Depicting the Stability of transethosome gel at Room Temperature.

\section{Stability Studies}

The refrigerated condition $\left(5 \pm 3^{\circ} \mathrm{C}\right)$ and $\left(25 \pm 2^{\circ} \mathrm{C}\right)$ for 28 days was used to study the stability of developed gel formulation. The Transethosomal gel was evaluated for drug content and EE at an interval of 7 days for 28 days. A negligible loss of drug is seen in refrigerated condition and room temperature. There is very less change in the EE, which proves that the formulation was found to be stable. The results of the stability studies of the gel in both the temperature conditions are summarized in Figure 8 and 9 .

\section{CONCLUSION}

Tramadol hydrochloride is a opiate analgesic acts as a $\mu$ opioidreceptor.'The tramadolhydrochlorideencapsulated transethosomes were formulated for effective pain management by cold method with different lipoidal carrier systems. Total 12 formulations were prepared by using cold method. Formulations were designed using ethanol, edge activator and phospholipid. Span 20 and Cremophor EL-35 were used as edge activators, soya lecithin and $1-\alpha$ Phosphatidylcholine from egg yolk contributes to lipid carrier and ethanol as solvent system. The physical evaluation of pure drug and along with excipients has shown compatibility. The FTIR results reveal that there is no interaction between the drug and excipient of the formulations. The drug content of the transethosomal formulations was fit to be in the range of 91.91 to $95.7 \%$ and formulations F8 and F11 showed higher EE78.96\% and $79.34 \%$ than other batches. The formulation exhibits an average particle size in the range of $149.34 \mathrm{~nm}$ to $198.10 \mathrm{~nm}$. As the concentration of edge activator increases in the formulation shows decrease vesicular size. Formulation developed using soya lecithin exhibits an increased particle size than egg yolk formulations. Soya lecithin formulations are viscous than the $1-\alpha$ Phosphatidylcholine from egg yolk formulations. The zeta potential of the transethosomal formulations was found between the ranges of $-7.76 \mathrm{mV}$ to $-22.69 \mathrm{mV}$. The optimized formulation F11 shows -22 $\mathrm{mV}$ and proven to be sufficient stable. Formulation F11 comprises an optimum concentration of ethanol 30\% with optimum concentration of phospholipid 3\% with a suitable edge activator (Cremophor EL-35) has found to be the optimum batch which exhibits a controlled rate of release of tramadol hydrochloride of $79.98 \%$ at the $8^{\text {th }} \mathrm{hr}$. The release pattern of the formulation F11 exhibit best fit of highest correlation coefficient by the First order kinetics. The gel formulation was studied for viscosity, drug content and EE. The gel characteristics were found to be satisfactory. The stability studies given away that the formulation was stable at refrigerated conditions $\left(5 \pm 3^{\circ} \mathrm{C}\right)$ for 28 days. Therefore, the study proves that transethosomal gel is possible carrier for the topical delivery of tramadol hydrochloride. These afford another route for the administration of the drug to conquer the reduced bioavailability and rigorous side effects connected with the oral route.

\section{ACKNOWLEDGEMENT}

We are highly indebted to Aurobindo Pharma Pvt Limited, Hyderabad for providing the gift sample of tramadol hydrochloride. The authors are grateful to the management of GIET School of Pharmacy, Rajahmundry, for facilitating our research work.

\section{CONFLICT OF INTEREST}

The authors declare no conflict of interest. 


\section{ABBREVIATIONS}

TEs: Transethosomes; TEM: Transmission electron microscopy; Rpm: Revolutions per minute; ${ }^{\circ} \mathbf{C}$ : Degree Celsius; mg: Milligram; FTIR: Fourier transform infrared spectroscopy; EE: Entrapment efficiency.

\section{REFERENCES}

1. Bhadra D, Jain NK, Umamaheshwari RB, Jain S. Ethosomes: A novel vesicular carrier for enhanced transdermal delivery of an anti HIV agent. Indian J Pharm Sci. 2004;66(1):72-81.

2. Ascenso A, Raposo S, Batista C, et al. Development characterization and skin delivery studies of related ultradeformable vesicles: Transfersomes, ethosomes and transethosomes. Int J Nanomedicine. 2015;10:5837-51.

3. Touitou E, Godin B, Weiss C. Enhanced delivery of drugs into and across the skin by ethosomal carriers. Drug Dev Res. 2000;50(3-4):406-15.

4. Akhtar N. Vesicles: A recently developed novel carrier for enhanced topical drug delivery. Curr Drug Deliv. 2014;11(1):87-97.

5. Abdulbaqi IM, Darwis $Y$, Khan NA, Assi RA, Khan AA. Ethosomal nano carriers: The impact of constituents and formulation techniques on ethosomal properties in vivo studies and clinical trials. Int J Nanomedicine. 2016;11:2279-304.

6. Song CK, Balakrishnan P, Shim CK, Chung SJ, Chong S, Kim DD. A novel vesicular carrier transethosome for enhanced skin delivery of voriconazole: Characterization and in vitrolin vivo evaluation. Colloids Surf B Biointerfaces. 2012;92:299-304.

7. Ainbinder D, Touitou E. A new approach for skin tumor treatment: From delivery system characterization to in vivo evaluation. Drug Deliv Transl Res. 2011;1(1):53-65.

8. Meng S, Chen Z, Yang L, et al. Enhanced transdermal bioavailability of testosterone propionate via surfactant-modified ethosomes. Int $\mathrm{J}$ Nanomedicine. 2013;8:3051-60.

9. Chung KS, Prabagar B, Chang-Koo S, Suk-Jae C, Saeho C, Dae-Duk K. A novel vesicular carrier transethosome for enhanced skin delivery of voriconazole: Characterization and in vitrolin vivo evaluation. Colloids and Surfaces B: Biointerfaces. 2012;92:299-304.

10. Chen ZX, Li B, Liu T, Wang X, Zhu Y, Wang L, et al. Sun: Evaluation of paeonol-loaded transethosomes as transdermal delivery carriers. Eur $\mathrm{J}$ Pharm Sci. 2017;99:240-5.

11. Kumari S, Pathak K. Cavamax W7 Composite psoralen ethosomal gel versus Cava max W7 psoralen solid complex gel for topical delivery: A comparative evaluation. Int J Pharm Investig. 2013;3(4):171-82.
12. Verma P, Pathak K. Nanosized ethanolic vesicles loaded with econazole nitrate for the treatment of deep fungal infections through topical gel formulation. Nanomedicine. 2012;8(4):489-96.

13. Jing Li, Xuling W, Ting Z, Chunling $W$, Zhenjun $\mathrm{H}$, Xiang L, et al. A review on phospholipids and their main applications in drug delivery systems. Asian J Pharm. 2015;10(2):81-98.

14. Maheshwari RG, Tekade RK, Sharma PA, et al. Ethosomes and ultradeformable liposomes for transdermal delivery of clotrimazole: A comparative assessment. Saudi Pharm J. 2012;20(2):161-70.

15. Lalit K, Shivani V, Kuljit S, DeoNandan P, Amit KJ. Ethanol Based Vesicular Carriers in Transdermal Drug Delivery: Nanoethosomes and Transethosomes in Focus. Nano World Journal. 2016;2(3):41-51.

16. Ibrahim MA, Yusrida D, Reem AA, Nurzalina AKK. Transethosome gels as carriers for the transdermal delivery of Colchicine optimization characterization and ex vivo evaluation. Drug Des Dev Ther. 2018;12:795-813.

17. Raza K, Singh B, Mahajan A, Negi P, Bhatia A, Katare OP. Design and evaluation of flexible membrane vesicles (FMVs) for enhanced topical delivery of capsaicin. J Drug Target. 2011;19(4):293-302.

18. Anil RP, Pravin DC, Ashwini BN. Oral Bioavailability Enhancement of Sertraline Hydrochloride by Nanoprecipitation and Solvent Diffusion Techniques for Stable Nanosuspension. Asian J Pharm. 2016;10(3):251-7.

19. Gondkar SB, Patil NR, Saudagar RB. Formulation Development and Characterization of Drug Loaded Transethosomes for Transdermal delivery. Int J Chemtech Res. 2017;10(6):535-44.

20. Varun G, Harmananpreet S, Amit B, Kaisar R, Sachin KS, Bhupinder S, et al. Systemic Development of transethosomal gel system of piroxicam: Formulation Optimization, in vitro Evaluation and ex vivo Assessment. APPS Pharm Sci Tech. 2016;18(1):58-71.

21. Akhtar N, Pathak K. Cavamax W. Composite ethosomal gel of clotrimazole for improved topical delivery: Development and comparison with ethosomal gel. AAPS Pharm Sci Tech. 2012;13(1):344-55.

22. Limsuwan T, Amnuaikit T. Development of ethosomes containing mycophenolic acid. Procedia Chem. 2012;4:328-35.

23. Ibrahim MA, Yusrida D, Nurzalina A, Reem AA, Arshad AK. Ethosomal snano carriers: The impact of constituents and formulation techniques on ethosomal properties in vivo studies and clinical trials. Int J Nanomedicine. 2016;11:2279-304

24. Neelam I, Dinesh K. Design development and evaluation of ethosomal gel of fluconazole for topical fungal infection. Int Jouof Engg Sci Invention Res and Dev. 2015;1(8):208-306.

25. Mistry A, Ravi KP, Pathare S. Ethosome: Unique Elastic Vesicular CarrierOverview. Int J Pharm. 2015;6(10):4129-36.

26. Sirisha M, Nazia B. Formulation and evaluation of topical and ethosomal gels of fluconazole and chlorhexidine BP. World Jou of Pharm and Pharm Sci. 2016;5(12):702-18.

\section{SUMMARY}

- The tramadol hydrochloride encapsulated transethosomes were formulated for effective pain management by cold method using different lipoidal carrier systems. Tramadol hydrochloride is a centrally acting opiate analgesic. Topically applied opioid predominately have central effects which plays a primary role on peripheral nervous system. The developed transethosomes were characterized for FTIR, drug content, EE, particle size, zeta potential, TEM, in vitro drug release and release kinetics.

- The in vitro release of the transethosomes was extensively studied and the effect of the edge activator, ethanol and phospholipid concentration on release studies was estimated by using Franz diffusion cell. From in vitro studies it was concluded that the release of tramadol hydrochloride was influenced by changing the ratio of phospholipid and ethanol. The optimised formulation was considered for topical gel preparation. 


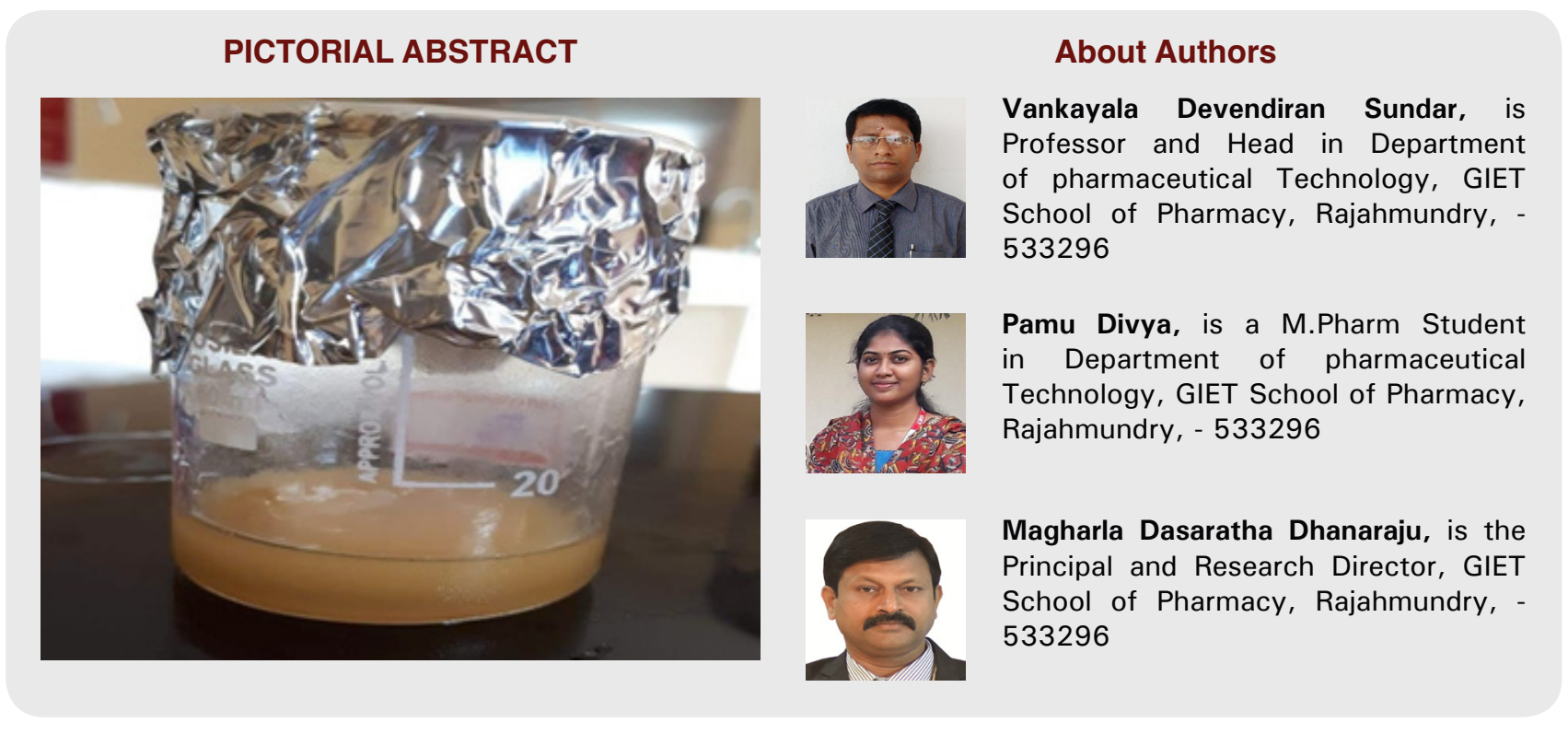

Cite this article: Sundar VD, Divya P, Dhanaraju MD. Design Development and Characterisation of Tramadol Hydrochloride Loaded Transethosomal Gel Formulation for Effective Pain Management. Indian J of Pharmaceutical Education and Research. 2020;54(2s):s88-s97. 